\title{
Up-Regulation of PTHrP and Bcl-2 Expression Characterizes the Progression of Osteochondroma towards Peripheral Chondrosarcoma and Is a Late Event in Central Chondrosarcoma
}

\author{
Judith V.M.G. Bovée, Lambert J.C.M. van den Broek, Anne-Marie Cleton-Jansen, \\ and Pancras C.W. Hogendoorn
}

Department of Pathology, Leiden University Medical Center, Leiden, The Netherlands

\begin{abstract}
SUMMARY: Chondrosarcomas are malignant cartilage-forming tumors arising centrally in bone (central chondrosarcoma) or within the cartilaginous cap of osteochondroma (peripheral chondrosarcoma). For hereditary multiple osteochondromas, two responsible genes, EXT1 and EXT2, have been cloned. Their recently elucidated role in heparan sulfate biosynthesis and Hedgehog diffusion leads to the hypothesis that EXT inactivation affects fibroblast growth factor (FGF) and Indian Hedgehog $(\mathrm{IHh}) /$ parathyroid hormone-related peptide (PTHrP) signaling, two important pathways in chondrocyte proliferation and differentiation. The expression of PTHrP, PTHrP-receptor, Bcl-2, FGF2, FGFR1, FGFR3, and p21 is investigated by immunohistochemistry in osteochondromas $(n=24)$ and peripheral $(n=29)$ and central $(n=20)$ chondrosarcomas. IHh/PTHrP and FGF signaling molecules are mostly absent in osteochondromas. Although no somatic EXT mutations were found in sporadic osteochondromas, the putative EXT downstream targets are affected similarly in sporadic and hereditary tumors. In chondrosarcomas, re-expression of FGF2, FGFR1, PTHrP, Bcl-2, and p21 is found. Expression levels increase with increasing histological grade. Up-regulation of PTHrP and Bcl-2 characterizes malignant transformation of osteochondroma because PTHrP and Bcl-2 expression is significantly higher in borderline and grade I peripheral chondrosarcomas compared with osteochondromas. In contrast, up-regulation of PTHrP and $\mathrm{Bcl}-2$ seems to be a late event in central cartilaginous tumorigenesis because expression is mainly restricted to high-grade central tumors. (Lab Invest 2000, 80:1925-1933).
\end{abstract}

C hondrosarcoma of bone is a malignant tumor characterized by the formation of cartilage and occurring principally in adults in the third to sixth decade. It is the second most frequent primary malignant bone tumor after osteosarcoma. The majority of chondrosarcomas (75\%) are located within the medullar cavity (central chondrosarcoma), whereas 15\% develop within the cartilage cap of a pre-existing osteochondroma (peripheral chondrosarcoma) (Mulder et al, 1993; Springfield et al, 1996). Although there are no apparent cytonuclear differences between central and peripheral chondrosarcomas, we previously demonstrated different genetic mechanisms to be operative in the origins of central and peripheral chondrosarcomas (Bovée et al, 1999a). Both subtypes are histologically classified into three grades, correlating with prognosis (Evans et al, 1977). Recurrent chondrosarcomas may exhibit a higher grade of malignancy than the original neoplasm (Bjornsson et al, 1998; Evans et al, 1977), suggesting that tumors may progress in grade.

Received September 12, 2000

Address reprint requests to: Dr. Judith V.M.G. Bovée, Department of Pathology, Leiden University Medical Center, P.O. box 9600, L1-Q, 2300 RC Leiden, The Netherlands.E-mail: J.V.M.G.Bovee@lumc.nl
Malignant transformation of solitary osteochondromas is low $(<1 \%)$, whereas it is estimated to occur in $1 \%$ to $5 \%$ of cases of hereditary multiple exostoses (HME), a familial skeletal disorder with an autosomal dominant mode of inheritance (Schmale et al, 1994; Wicklund et al, 1995). HME is genetically heterogeneous, and at present, two responsible genes, EXT1 and EXT2, located respectively at 8q24 and 11p11p12, have been isolated (Ahn et al, 1995; Stickens et al, 1996; Wuyts et al, 1996). We have previously demonstrated EXT1 to be operative as a tumorsuppressor gene during hereditary osteochondroma development according to Knudson's two-hit model (Bovée et al, 1999b). Surprisingly, no somatic EXT1 cDNA alterations have been found in sporadic tumors (Bovée et al, 1999b).

The human EXT1 and EXT2 genes both encode endoplasmic reticulum-localized type II transmembrane glycoproteins that possess or are tightly associated with glycosyltransferase activities involved in heparan sulfate polymerization (Lind et al, 1998; McCormick et al, 1998; Simmons et al, 1999). It was recently suggested that EXTL2, a related protein, is the key enzyme for the chain initiation of heparan sulfate (Kitagawa et al, 1999), after which an EXT1/ EXT2 complex performs chain polymerization (Kitagawa et al, 1999; McCormick et al, 1999, 2000). 
Heparan sulfate proteoglycans (HSPG) are large macromolecules composed of heparan sulfate glycosaminoglycan chains linked to a protein core, which participate in cell signaling pathways (McCormick et al, 1999). For instance, HSPGs are required for highaffinity interactions of fibroblast growth factor (FGF) with its receptor (FGFR) (Erlebacher et al, 1995; Goldfarb, 1996). Furthermore, an EXT1 homolog (tout-velu, Ttv) in drosophila, which is also involved in heparan sulfate biosynthesis, is required for diffusion of an important segment-polarity protein called Hedgehog (Hh), a homolog of mammalian Indian Hedgehog (IHh) (Bellaiche et al, 1998; The et al, 1999; Toyoda et al, 2000).

The orderly proliferation and maturation of chondrocytes in longitudinal columns within the normal growth plate (Fig. 1A) is regulated by a delicate paracrine feedback loop involving both IHh and FGF signaling (Fig. 1, B and C) (van der Eerden et al, 2000). Chondrocytes in transition from the proliferative to the hypertrophic zone start to secrete IHh, which binds to its receptor, Patched (Ptc). This results in increased secretion of parathyroid hormone-related peptide (PTHrP) via an as yet incompletely resolved mechanism. Binding of PTHrP to its receptor at the late-proliferating chondrocytes (Erlebacher et al, 1995) inhibits further differentiation by a direct or indirect up-regulation of the anti-apoptotic protein Bcl-2. This results in fewer IHh-producing cells, which closes the feedback loop (Amling et al, 1997). Thus, PTHrP regulates the pace of chondrocyte differentiation by delaying the progression of chondrocytes towards the hypertro- phic zone, allowing longitudinal bone growth (van der Eerden et al, 2000).

IHh signaling is repressed when the FGF-receptor 3 (FGFR3), which is expressed at the proliferative zone of the growth plate, is activated by a presently unknown ligand (Erlebacher et al, 1995; Goldfarb, 1996; Naski et al, 1998). Furthermore, FGFR3 inhibits chondrocyte proliferation via induction of expression of p21 WAF1/CIP1, which is an inhibitor of the cell cycle (Sahni et al, 1999). In contrast to FGFR3, FGF2 (basic FGF) is the most potent mitogen for chondrocytes in vitro and stimulates extracellular matrix synthesis via its receptor (eg, FGF-receptor 1) (Iwamoto et al, 1991; Kato and Iwamoto 1990).

The stratified zones of the growth plate are less well regulated but can sometimes still be recognized in osteochondroma. The recent elucidation of the function of the EXT genes led us to the hypothesis that inactivation of both copies of EXT(1) in normal cells of the growth plate (Bovée et al, 1999b) will result in altered heparan sulfate expression at the cell surface of chondrocytes (McCormick et al, 1998), which will in turn affect FGF signaling by preventing high-affinity binding of FGF to its receptor (Erlebacher et al, 1995; Goldfarb, 1996). Furthermore, because the EXT1 homolog in drosophila is required for diffusion of Hedgehog (Bellaiche et al, 1998; The et al, 1999; Toyoda et al, 2000), EXT1 inactivation in humans may also affect $\mathrm{IHh} / \mathrm{PTHrP}$ signaling by preventing the diffusion of $\mathrm{IHh}$. We have therefore investigated the protein expression of these putative downstream effectors of EXT by immunohistochemistry in a well-characterized group of osteochondromas and chondrosarcomas.

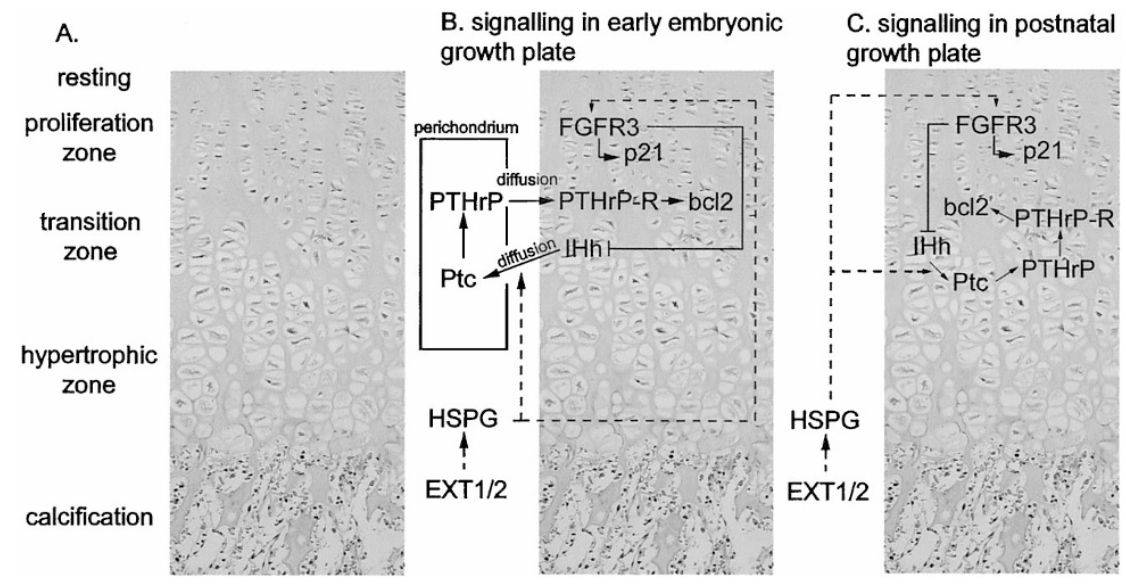

Figure 1.

$A$, Light-micrograph of a normal postnatal growth plate that is characterized by the orderly proliferation and maturation of chondrocytes in longitudinal columns forming stratified zones of resting, proliferative, prehypertrophic/maturing, and hypertrophic cartilage. Ossification begins with invasion of calcified hypertrophic cartilage by capillaries accompanied by apoptosis of terminal hypertrophic chondrocytes, resorption of cartilage matrix, and deposition of bone matrix by osteoblasts. $B$, Growth signaling in the early embryonic growth plate is depicted within the photograph. Chondrocytes in the transition zone secrete Indian hedgehog (IHh), which has to diffuse to the lateral perichondrium where its receptor, Patched (Ptc), is expressed. Binding will induce up-regulation of parathyroid hormone-related peptide $(\mathrm{PTHrP})$ at the apical perichondrium, which then diffuses to its receptor localized at late-proliferating chondrocytes. Via up-regulation of Bcl-2, further differentiation of late proliferating chondrocytes is inhibited, resulting in fewer IHh-producing cells, which closes the feedback loop. Activation of fibroblast growth factor receptor-3 (FGFR3), expressed at the proliferative zone, by an as yet unknown ligand inhibits chondrocyte proliferation via up-regulation of p21WAF1/CIP1 and represses IHh signaling. C, Growth signaling in the postnatal growth plate. The IHh/PTHrP feedback loop is now confined to the growth plate. IHh will bind Ptc in the hypertrophic zone, stimulating PTHrP expression. PTHrP then binds its receptor in the prehypertrophic zone, up-regulating Bcl-2. Defective or absent EXT proteins leading to altered or absent heparan sulfate proteoglycans (HSPG) expression at the cell surface may affect high-affinity binding at the FGFR3 receptor and may disturb IHh diffusion towards the apical perichondrium (early embryonic growth plate) or to its neighboring cells (postnatal growth plate). 


\section{Results}

\section{Immunohistochemistry}

Expression of FGF2, FGFR1, p21, PTHrP, and Bcl-2 was found in $25 \%$ to $83 \%$ chondrosarcomas. In contrast, only $0 \%$ to $41 \%$ of osteochondromas expressed these antigens (Table 1). Examples are shown in Figures 2 and 3 . To the extent that the stratified zones that are found in the normal growth plate could still be recognized in positive osteochondroma cases, no spatial distribution of protein expression was found. The expression of the PTHrP-receptor and FGF-receptor 3 was almost equally distributed over osteochondromas and chondrosarcomas.

Some immunostained sections could not be evaluated because of the loss of attachment to the glass slides or the absence of staining of an internal positive control. In the latter situation, prolonged decalcification may have altered the configuration of the antigen, and these cases were therefore excluded from the analysis. This was mainly a problem in osteochondromas containing a lot of bony material requiring long decalcification. Results of all cases that could be evaluated are summarized in Table 1.

\section{Statistical Analysis}

Osteochondroma versus Peripheral Chondrosarcoma. The expression of FGF2, FGFR1, p21, PTHrP, and $\mathrm{Bcl}-2$ was found in a significantly higher percentage of peripheral chondrosarcomas compared with osteochondromas (Table 1). A further comparison between the group of osteochondromas and a group of exclusively borderline and grade I peripheral chondrosarcomas revealed a significantly higher percentage expressing PTHrP $(p=0.003)$ and $\mathrm{Bcl}-2$ ( $p=$ 0.001 ) (Fisher's Exact Test) in the latter group (borderline and grade I peripheral chondrosarcomas)(Fig. 3). PTHrP expression was found in 7 of 12 borderline and grade I chondrosarcomas, but in only 1 of 18 osteochondromas, resulting in a sensitivity of $58 \%$ and a specificity of $94 \%$. Five of 9 borderline and grade I chondrosarcomas expressed Bcl-2 compared with none of 19 osteochondromas, resulting in a sensitivity of $56 \%$ and a specificity of $100 \%$.

Peripheral versus Central Chondrosarcoma. Expression of PTHrP and Bcl-2 was also significantly higher in peripheral compared with central chondrosarcomas ( $p=0.031$ and $p=0.017$, respectively). Remarkably, in central chondrosarcomas, expression of PTHrP and $\mathrm{Bcl}-2$ was mainly restricted to high-grade cases (Table 2, Fig. 3), with the exception that one borderline central chondrosarcoma expressed both proteins.

Sporadic versus Hereditary Cases. There were no differences in expression of any molecule tested between HME and non-HME peripheral tumors (Fisher's Exact test). Considering the level of expression, however, only $\mathrm{Bcl}-2$ expression was stronger in non-HME cases compared with HME cases $(p=0.031$, Chisquare test, linear by linear). For the level of expression of the other factors, no differences were found. Furthermore, within the group of osteochondromas, there were no differences in expression between patients before and after the age of 15 years or between male and female patients.

Correlation with Prognosis. The expression levels of FGF2 $(p=0.039)$, FGFR1 $(p=0.000)$, FGFR3 $(p=$ $0.043), \mathrm{p} 21(p=0.002), \mathrm{PTHrP}(p=0.039)$, and Bcl-2 $(p=0.011)$ increased with increasing histological grade (Chi square test, linear by linear). Only the expression of p21 was correlated with a shorter disease-free survival ( $p=0.0198$, Log Rank Test), although the effect of this parameter on disease-free survival was not independent of histological grading $p$ $=0.2443, \mathrm{p} 21 ; p=0.0520$, histological grade, Cox regression analysis).

Correlation between the Expression of the Different Proteins. Considering the delicate paracrine growth signaling in which the investigated molecules are normally involved (Fig. 1), a correlation between the level of expression of the separate molecules investigated was tested using the Chi square test, linear by linear. Indeed, for most proteins the level of expression strongly correlated with the level of expression of

Table 1. Results of Immunohistochemistry

\begin{tabular}{|c|c|c|c|c|c|c|c|c|}
\hline \multirow[b]{2}{*}{ Antigen } & \multicolumn{2}{|c|}{ Osteochondroma } & \multirow{2}{*}{$\begin{array}{c}\text { Osteochondroma vs } \\
\text { peripheral CS } \\
\text { p value }{ }^{b}\end{array}$} & \multicolumn{2}{|c|}{ Peripheral CS } & \multirow{2}{*}{$\begin{array}{l}\text { Peripheral vs } \\
\text { central CS } \\
\text { p value }^{b}\end{array}$} & \multicolumn{2}{|c|}{ Central CS } \\
\hline & $\operatorname{pos}^{a}$ & $\%$ & & pos & $\%$ & & pos & $\%$ \\
\hline FGF-2 & $2 / 17$ & 12 & $p=0.044$ & $12 / 27$ & 44 & $p=0.767$ & 9/18 & $50 \%$ \\
\hline FGFR-1 & $9 / 22$ & 41 & $p=0.017$ & $21 / 27$ & 78 & $p=0.721$ & $15 / 18$ & $83 \%$ \\
\hline FGFR-3 & $4 / 21$ & 19 & $p=0.517$ & 8/28 & 29 & $p=0.737$ & 4/19 & $21 \%$ \\
\hline p21 & $5 / 20$ & 25 & $p=0.009$ & $17 / 26$ & 65 & $p=0.539$ & $10 / 19$ & $53 \%$ \\
\hline PTHrP & $1 / 18$ & 6 & $p=0.000$ & $23 / 29$ & 79 & $p=0.031$ & 9/19 & $47 \%$ \\
\hline PTHrP-R & $6 / 19$ & 32 & $p=0.543$ & $11 / 26$ & 42 & $p=0.540$ & $6 / 20$ & $30 \%$ \\
\hline Bcl-2 & $0 / 19$ & 0 & $p=0.000$ & $15 / 24$ & 63 & $p=0.017$ & $5 / 20$ & $25 \%$ \\
\hline
\end{tabular}

FGF-2, fibroblast growth factor 2; FGFR-1, fibroblast growth factor receptor 1; PTHrP, parathyroid hormone-related peptide; PTHrP-R, PTHrP receptor.

Results of the immunohistochemical analysis are shown for osteochondromas, peripheral chondrosarcomas (CS), and central chondrosarcomas.

${ }^{a}$ pos: the number of positive tumors/total number of tumors that could be evaluated.

${ }^{b}$ Results within the group of osteochondromas are compared to results within the group of peripheral chondrosarcomas for each antigen tested using the Fisher's Exact Test. Similarly, results within the group of peripheral chondrosarcomas are compared to the results within the group of central chondrosarcomas. Significant $\mathrm{p}$ values are shown in bold. For PTHrP and $\mathrm{Bcl}-2$, differing in expression between central and peripheral chondrosarcomas, results per tumor grade are shown in Table 2. 


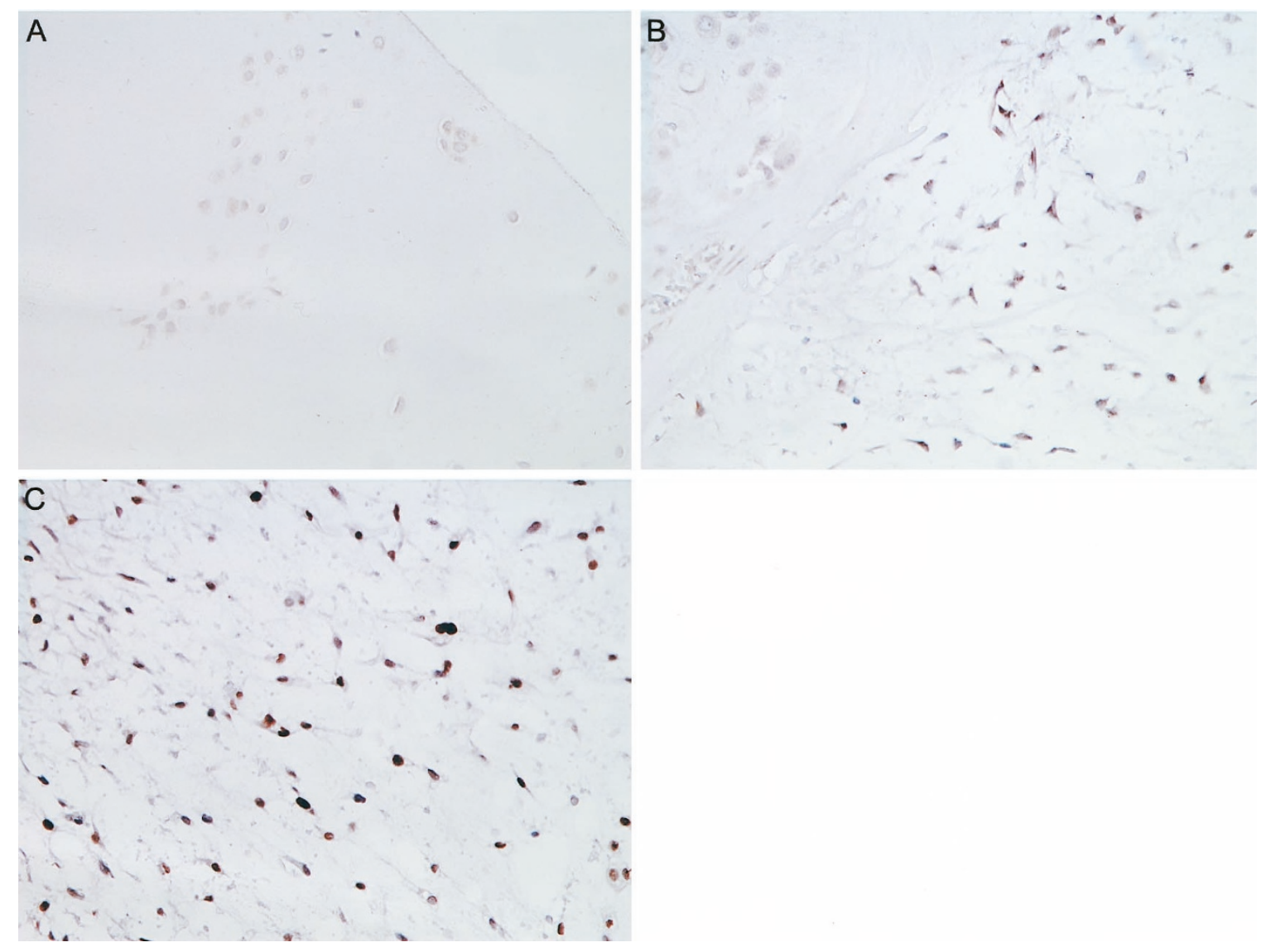

Figure 2.

A, FGFR1 expression within osteochondroma. The remaining cartilaginous cap (not shown) has low cellularity without FGFR1 expression, whereas this part of the cartilaginous cap, with increased cellularity, demonstrates FGFR1 expression, suggesting local progression. $B$, Grade III peripheral chondrosarcoma demonstrating cytoplasmic FGFR3 expression with moderate intensity, found in $50 \%$ to $75 \%$ of the tumor cells. C, Grade III peripheral chondrosarcoma demonstrating strong nuclear p21 expression, found in $50 \%$ to $75 \%$ of the tumor cells.

other proteins. For instance, strong Bcl-2 expression was correlated with a strong expression of all other molecules ( $p$ value range: $0.000-0.007$, Chi-square test, linear by linear). A correlation was lacking only between FGF2 and p21 ( $p=0.051)$, FGFR3 and p21 $p$ $=0.394)$, PTHrP and PTHrP-R $(p=0.249)$, and FGFR3 and PTHrP-R $(p=0.069)$.

\section{Discussion}

We studied the expression of seven proteins that are known to be involved in normal chondrocyte growth and differentiation (Fig. 1) and that are predicted to be affected by EXT inactivation in cartilaginous tumors. We previously demonstrated inactivation of both copies of EXT1 in hereditary osteochondromas (Bovée et al, 1999b). The involvement of EXT1 and EXT2 proteins in heparan sulfate biosynthesis (McCormick et al, 1998) and Hedgehog diffusion (Bellaiche et al, 1998) was shown recently. This leads to the hypothesis that EXT inactivation will affect both IHh/PTHrP signaling by preventing the diffusion of $\mathrm{IHh}$ (Bellaiche et al, 1998; The et al, 1999; Toyoda et al, 2000) and FGF signaling by preventing HSPG-mediated high-affinity binding of FGF to its receptor (Erlebacher et al, 1995; Goldfarb, 1996) (Fig. 1).
Table 2. Results of Bcl-2 and PTHrP Immunohistochemistry in Chondrosarcomas, According to Histological Grade

\begin{tabular}{llrlrr}
\hline & \multicolumn{2}{c}{ Peripheral CS } & & \multicolumn{2}{c}{ Central CS } \\
\cline { 2 - 3 } & pos $^{a}$ & $\%$ & & pos $^{a}$ & $\%$ \\
\hline PTHrP & & & & \\
Borderline & $0 / 2$ & 0 & & $1 / 3$ & 33 \\
Grade I & $7 / 10$ & 70 & & $4 / 9$ & 44 \\
Grade II & $9 / 10$ & 90 & & $3 / 5$ & 60 \\
Grade III & $7 / 7$ & 100 & & $1 / 1$ & 100 \\
Metastasis & - & - & & $0 / 1$ & 0 \\
Bcl-2 & & & & \\
Borderline & $0 / 1$ & 0 & & $1 / 3$ & 33 \\
Grade I & $5 / 8$ & 63 & & $0 / 9$ & 0 \\
Grade II & $5 / 8$ & 63 & & $2 / 6$ & 33 \\
Grade III & $5 / 7$ & 71 & & $1 / 1$ & 100 \\
Metastasis & - & - & & $1 / 1$ & 100 \\
\hline
\end{tabular}

a pos: the number of positive tumors/total number of tumors that could be evaluated.

We demonstrate that the growth regulators involved in both IHh/PTHrP and FGF signaling are absent, or only lowly expressed in a minority of cells, in osteo- 

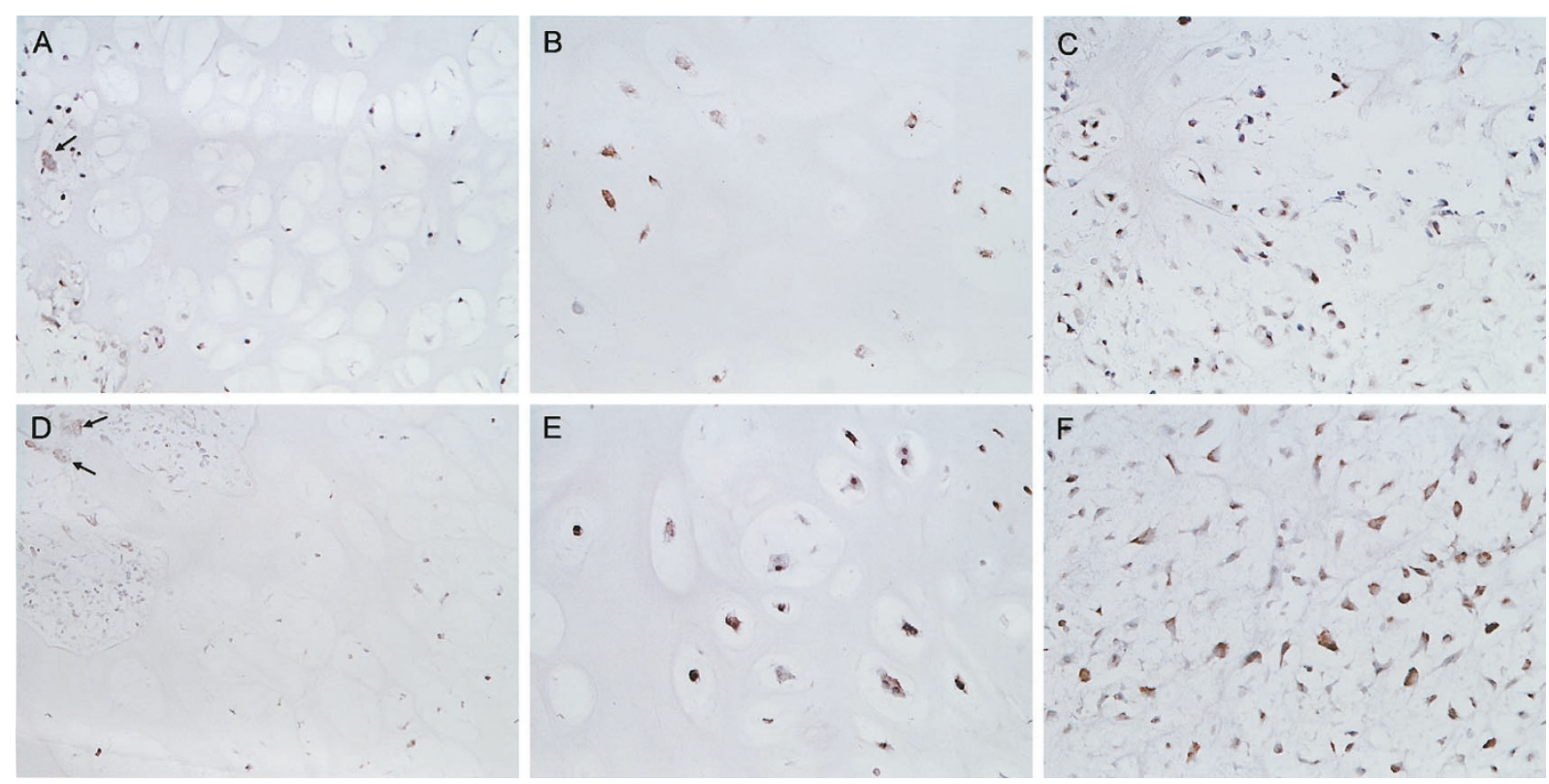

\section{Figure 3.}

A, Low-power view of a sporadic osteochondroma demonstrating weak PTHrP expression found in only $1 \%$ to $25 \%$ of the tumor cells, which is therefore scored as negative. Note the strong staining of the osteoclast, used as an internal positive control. B, Grade I peripheral chondrosarcoma secondary to a sporadic osteochondroma demonstrating PTHrP expression with moderate intensity, found in $25 \%$ to $50 \%$ of the tumor cells. C, In central chondrosarcoma, PTHrP expression was mainly restricted to high-grade tumors. A grade III central chondrosarcoma is shown with staining of moderate intensity, found in $50 \%$ to $75 \%$ of the tumor cells. $D$, Low-power view of an osteochondroma from a patient demonstrating hereditary multiple exostoses syndrome with weak Bcl-2 expression found in only $1 \%$ to $25 \%$ of the tumor cells, which is therefore scored as negative. Note strong positive staining of the osteoclast, used as internal positive control. $E$, Same grade I peripheral chondrosarcoma as shown in B, clearly demonstrating moderate Bcl-2 expression, found in $50 \%$ to $75 \%$ of the tumor cells. $F$, Same grade III central chondrosarcoma as shown in C, clearly demonstrating strong Bcl-2 positive staining in $75 \%$ to $100 \%$ of the tumor cells.

chondromas. Although both the PTHrP receptor and the FGF receptor 1 are detected in $32 \%$ and $41 \%$ of osteochondromas, respectively, their ligands PTHrP and FGF2 are detected in only $6 \%$ and $12 \%$ of osteochondromas, respectively. Others demonstrate the absence of FGF-2 expression in enchondromas, whereas all chondrosarcomas studied are positive with varying intensity (Uria et al, 1998). Investigations in drosophila demonstrate that defects in the EXT1 homolog tout velu specifically affect Hedgehog diffusion, whereas other HSPG-dependent pathways, such as FGF or Wingless signaling, are unaffected (The et al, 1999). Our data however suggest that in human osteochondromas, IHh/PTHrP and FGF signaling are affected equally, and often simultaneously. Alternatively, because osteochondromas cease growing when the growth plates close, it may be that with puberty most of these growth-signaling molecules are down-regulated in osteochondroma in a physiological manner under hormonal control. However, we did not find differences in (the level of) expression of all factors in osteochondromas before and after the age of 15 .

In a previous study, we failed to detect any somatic mutations in EXT1 or EXT2 in sporadic osteochondromas and chondrosarcomas, despite loss of heterozygosity (LOH) at the EXT1 locus at 8q24 (Bovée et al, 1999b). So far, only one somatic EXT1 mutation in a sporadic chondrosarcoma has been described in the literature (Hecht et al, 1997). These data may imply that another mechanism is operative in sporadic compared with hereditary osteochondroma development. In the present study, we show, however, that the putative downstream effectors of EXT are affected similarly in sporadic and hereditary osteochondromas and peripheral chondrosarcomas, because we did not find any differences in expression. This suggests that, in sporadic osteochondroma development, other genetic aberrations affecting the same EXT pathway are operative.

Interestingly, re-expression of most signaling molecules in chondrosarcomas is demonstrated, because the expression of FGF2, FGFR1, p21, PTHrP, and $\mathrm{Bcl}-2$ is significantly higher in peripheral chondrosarcomas compared with osteochondromas. Furthermore, the levels of expression of all factors, apart from the PTHrP receptor, increase with increasing histological grade. This re-expression of growth factors normally involved in cartilage growth and differentiation is probably responsible for the proliferation and cell survival in chondrosarcomas. Whether this reexpression after malignant transformation is EXTdependent or not remains to be elucidated. However, because of the fact that in hereditary osteochondromas EXT is inactivated irreversibly (Bovée et al, $1999 b)$, the re-expression is most probably induced by other signals. Perhaps, growth stimulation becomes autocrine instead of paracrine, avoiding extracellular HSPG-dependent signaling.

The distinction between benign tumors and those of low-grade malignancy is considered difficult at both the clinico-radiological (Geirnaerdt et al, 1997) and histological levels (Mirra et al, 1985), and so far the diagnosis is usually based on a combination of clinical, radiological, and histological findings. We demon- 
strate expression of PTHrP and $\mathrm{Bcl}-2$ in a significantly higher percentage of borderline/grade I peripheral chondrosarcomas compared with osteochondromas. This suggests that up-regulation of the expression of these two proteins characterizes malignant transformation of osteochondroma. Although the sensitivity is relatively low (58\% and $56 \%$ ), a specificity of $94 \%$ (PTHrP) and 100\% (Bcl-2) is found because expression, especially of $\mathrm{Bcl}-2$, is mostly absent in osteochondromas. Positive $\mathrm{Bcl}-2$ immunostaining seems, therefore, to be indicative of malignancy. Additional studies, including double stainings and other methodologies like mRNA in situ hybridization, should be performed on a larger series of osteochondromas and low-grade chondrosarcomas to determine whether the detection of Bcl-2 and/or PTHrP can be used in the differential diagnosis between benign and lowgrade malignant peripheral chondrosarcomas in the future.

We previously demonstrated a diverging genetic mechanism to be operative in the origin of central and peripheral chondrosarcoma (Bovée et al, 1999a). Peripheral chondrosarcomas are characterized by genetic instability as demonstrated by a broad range in DNA ploidy and a high percentage of loss of heterozygosity involving many different chromosomes. In contrast, central chondrosarcomas are mostly peridiploid and have limited LOH (Bovée et al, 1999a). In the present study, expression of PTHrP and $\mathrm{Bcl}-2$ was found in a significantly higher percentage of peripheral chondrosarcomas compared with central chondrosarcomas, suggesting that up-regulation of these two proteins is highly specific for malignant transformation during peripheral cartilaginous tumorigenesis. In contrast, in central chondrosarcoma, up-regulation of PTHrP and Bcl-2 seems to be a late event, because expression is mainly found in high-grade tumors. Amling et al (1998) reported a high co-expression of PTHrP and Bcl-2 only in grade II and grade III chondrosarcomas without subdivision in central and peripheral chondrosarcoma. These data suggest that, in central cartilaginous tumorigenesis, up-regulation of $\mathrm{PTHrP}$ and $\mathrm{Bcl}-2$ is a late event characterizing the progression from low-grade towards high-grade chondrosarcoma. Thus, these data substantiate that different genetic pathways are involved in the genesis of central and peripheral chondrosarcoma.

In conclusion, we investigated the protein expression of molecules that are normally involved in chondrocyte proliferation and differentiation and that are predicted to be affected by EXT inactivation. Our data suggest that indeed both IHh/PTHrP and FGF signaling are affected during osteochondroma development. Although we previously failed to detect any somatic EXT mutations in sporadic osteochondroma, we here demonstrate that the putative EXT downstream targets are affected similarly in sporadic and hereditary peripheral tumors. In chondrosarcomas, re-expression of most signaling molecules is found, and although the up-regulation of PTHrP and $\mathrm{Bcl}-2$ seems to be a late event in central chondrosarcoma, malignant transformation of osteochondroma towards low-grade peripheral chondrosarcoma is characterized by the up-regulation of these two molecules.

\section{Materials and Methods}

\section{Patient Data}

Formalin-fixed, paraffin-embedded material was available for 73 tumors (osteochondroma, $n=24$; peripheral chondrosarcoma, $n=29$; and central chondrosarcoma, $n=20$ ) from 64 patients. Six hereditary osteochondroma cases were retrieved from other laboratories using PALGA (Dutch National Information System for Pathology, Utrecht, The Netherlands), whereas all other cases originated from the files of the Leiden University Medical Center (Leiden, The Netherlands), a tertiary bone tumor referral center. Dedifferentiated, mesenchymal, juxtacortical, and clear-cell chondrosarcomas were excluded because of their distinctly different clinico-pathological features. Pa-

Table 3. Clinical and Tumor-Related Data

\begin{tabular}{llll}
\hline & $\begin{array}{c}\text { Osteochondroma } \\
20 \text { patients } \\
(24 \text { tumors })\end{array}$ & $\begin{array}{l}\text { Peripheral CS } \\
26 \text { patients } \\
(29 \text { tumors })\end{array}$ & \multicolumn{1}{c}{$\begin{array}{c}\text { Central CS } \\
18 \text { patients } \\
\text { (20 tumors) }\end{array}$} \\
\hline Male/female & $15 / 5$ & $14 / 12$ & $6 / 12$ \\
Median age at diagnosis & $20.7 \mathrm{yr}$ & $39.8 \mathrm{yr}$ & $37.6 \mathrm{yr}$ \\
(range 4-49) & $($ range 16-68) & (range 14-63) \\
Histological grade & & 2 & 3 \\
Borderline & & 10 & 9 \\
Grade I & & 10 & 6 \\
Grade II & & 7 & 1 \\
Grade III & & $7 / 20^{a}$ & 1 \\
Metastasis & $11 / 18^{a}$ & 46 mo & - \\
Hereditary multiple exostoses & - & (range 13-128) & (range 16-192) \\
Median follow-up & & &
\end{tabular}

CS, chondrosarcoma.

a Patients with inconclusive data are omitted. 
tient data, shown in Table 3, were obtained by review of pathology specimens and reports, clinical charts, and radiographs. Central and peripheral chondrosarcoma were separated based upon accepted clinicopathological and radiological criteria (Huvos, 1991). For peripheral chondrosarcoma, a pre-existing osteochondroma or an identifiable stalk was documented either radiographically or by gross pathology. Multiplicity of osteochondromas, which was considered the criterion for HME because an unequivocal family history was not always available, could be assessed for 38 patients. Histological grading was performed according to the method of Evans et al (1977). Five tumors were diagnosed as borderline chondrosarcoma because they showed histological features that were not sufficient to diagnose grade I chondrosarcoma, although their X-rays showed features of an aggressive neoplasm (Unni, 1996).

\section{Antibodies and Controls}

Details of the available antibodies and controls used are described in Table 4. The commercially available antibodies against $\mathrm{IHh}$ and its receptor, Ptc (Santa Cruz Biotechnology, Santa Cruz, California), were shown not to work reliably in our hands, either on fresh-frozen or formalin-fixed, paraffin-embedded material, and were therefore not used in this study. The FGFR-1 antibody was kindly provided by Dr. J. Walters (Oxford Brookes University, Oxford, United Kingdom), and the characteristics of this antibody have been described (Bovée et al, 1998; De-Boer et al, 1996). To avoid false-negative results, all antibodies used were tested for their susceptibility to formalin fixation. Immunohistochemistry was performed on appropriate tissues fixed for 1, 3, 7, and 40 days, respectively. None of the antibodies revealed diminished staining reactivity or staining sensitivity. Moreover, for most of the antibodies used, internal positive controls were present in the histological slides (Hughes, 1997; Hughes and Hall, 1993; Qu et al, 1995; Reed et al, 1995) (Table 4) to determine whether negative tumor cells are truly negative or whether prolonged decalcification might have altered the configuration of the antigen, resulting in a negative internal control. As negative controls, slides were incubated with mouse or rabbit immunoglobulin $\mathrm{G}$ ( $\mathrm{lgG}$ ) of a corresponding (iso-)type and concentration instead of with primary specific antibodies.

\section{Immunohistochemistry}

Immunohistochemical reactions were performed according to standard laboratory methods as described previously (Bovée et al, 1998). After deparaffinizing, rehydration, and blocking of endogenous peroxidase, antigen retrieval was performed as detailed in Table 4, followed by an overnight incubation with the primary antibodies. Biotin-labeled rabbit antimouse or swine antirabbit immunoglobulins and a biotinylated HRPStreptavidin complex (DAKO, Glostrup, Denmark) were applied. Visualization was carried out in a diami-

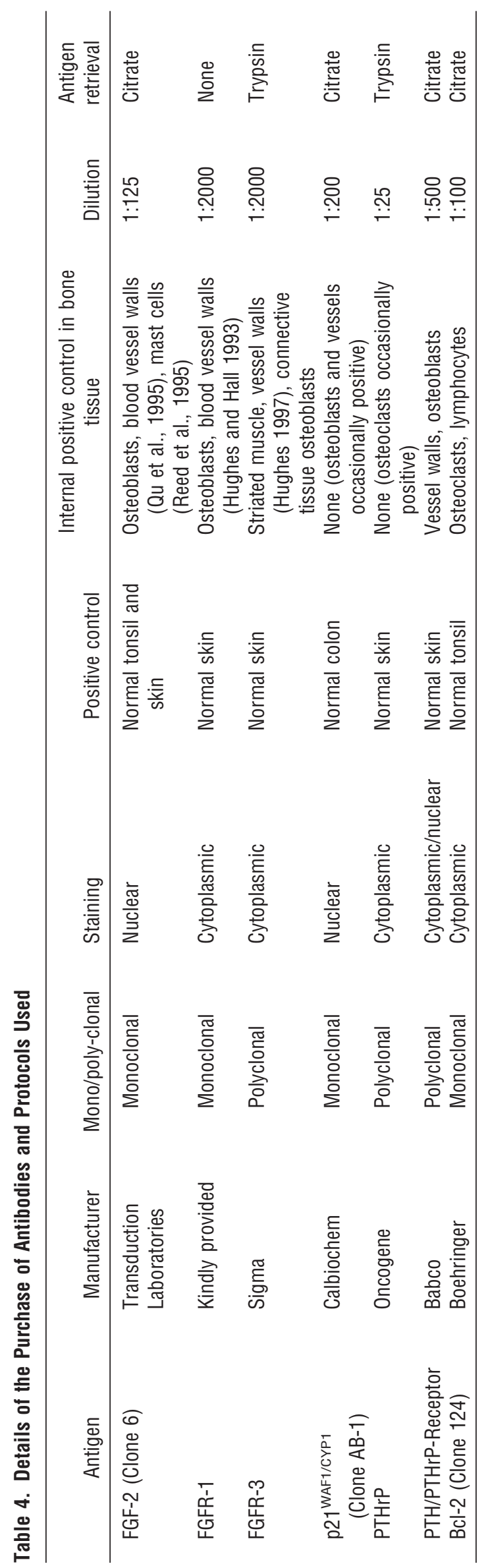

Laboratory Investigation • December $2000 \bullet$ Volume $80 \bullet$ Number 12 
nobenzidine solution (Sigma, St. Louis, Missouri) with the addition of $0.01 \mathrm{M}$ Imidazole for FGFR-1. Hematoxylin was used for counterstaining the slides.

\section{Evaluation and Scoring}

Staining was evaluated by two observers independently. Scoring was performed as described (Bovée et al, 1999a, 1999c; Detre et al, 1995; Elkhuizen et al, 1999) with some minor modifications. In brief, both staining intensity ( 0 , no staining; 1 , weak; 2 , moderate; 3 , strong intensity, as related to a positive internal control) and the percentage of positive tumor cells $(0,0 \% ; 1,1 \%$ to $24 \%$; 2, $25 \%$ to $49 \% ; 3,50 \%$ to $74 \% ; 4,75 \%$ to $100 \%$ positive tumor cells) were evaluated, and scores of intensity and percentage positivity were added up. In general, a score greater than 3 was considered positive (Elkhuizen et al, 1999), with the exception of p21 and FGFR1, for which a score greater than 0 was considered positive. For instance, for PTHrP and FGF2, almost all tumors demonstrated variable expression leading to a cut-off at a score greater than 3, whereas expression of p21 and FGFR1 was completely absent in about half of the tumors, allowing a cut-off at a score greater than 0 . The observers were blinded towards all clinicopathological data.

\section{Statistical Analysis}

Immunohistochemical results of peripheral chondrosarcomas were compared to osteochondromas as well as to central chondrosarcomas using Fisher's Exact test. In the same way, a comparison was made between the group of osteochondromas and a group of only borderline and grade I peripheral chondrosarcomas. Sensitivity and specificity were calculated for PTHrP and Bcl-2 to determine whether their expression could differentiate between osteochondroma and low-grade peripheral chondrosarcoma. Also, HME cases were compared to non-HME cases using Fisher's Exact Test. To test the prognostic value of the investigated molecules, a correlation between histological grade and the level of positivity was investigated using the Chi-square test, linear by linear. To test a correlation with disease-free survival, the Log Rank Test was performed for all variables separately. The Cox regression analysis was used to test whether certain parameters have an independent effect on disease-free survival. Correlation of the levels of expression of molecules with each other was tested using the Chi-square test, linear by linear. $p$ values equal to or less than 0.05 were considered to be significant.

\section{Acknowledgements}

The authors gratefully thank Dr. P. Eilers for advice on statistical analysis, Dr. J. Walters (Oxford Brookes University, Oxford, United Kingdom) for providing the FGFR-1 antibody, Mrs. M.A.N. Blonk-Beckers and Mr. B. van der Eerden for expert technical assistance, Dr. M. Karperien for fruitful discussions, and PALGA for the use of their database.

\section{References}

Ahn J, Ludecke H, Lindow S, Horton WA, Lee B, Wagner MJ, Horsthemke B, and Wells DE (1995). Cloning of the putative tumour suppressor gene for hereditary multiple exostoses (EXT1). Nat Genet 11:137-143.

Amling M, Neff L, Tanaka S, Inoue D, Kuida K, Weir E, Philbrick WM, Broadus AE, and Baron R (1997). Bcl-2 lies downstream of parathyroid hormone related peptide in a signalling pathway that regulates chondrocyte maturation during skeletal development. J Cell Biol 136:205-213.

Amling M, PosI M, Hentz MW, Priemel M, and Delling G (1998). PTHrP and Bcl-2: Essential regulatory molecules in chondrocyte differentiation and chondrogenic tumors. Verh Dtsch Ges Pathol 82:160-169.

Bellaiche Y, The I, and Perrimon N (1998). Tout-velu is a drosophila homologue of the putative tumour suppressor EXT1 and is needed for Hh diffusion. Nature 394:85-88.

Bjornsson J, McLeod RA, Unni KK, Ilstrup DM, and Pritchard DJ (1998). Primary chondrosarcoma of long bones and limb girdles. Cancer 83:2105-2119.

Bovée JVMG, Cleton-Jansen AM, Kuipers-Dijkshoorn N, Van den Broek LJCM, Taminiau AHM, Cornelisse CJ, and Hogendoorn PCW (1999a). Loss of heterozygosity and DNA ploidy point to a diverging genetic mechanism in the origin of peripheral and central chondrosarcoma. Genes Chromosomes Cancer 26:237-246.

Bovée JVMG, Cleton-Jansen AM, Wuyts W, Caethoven G, Taminiau AHM, Bakker E, Van Hul W, Cornelisse CJ, and Hogendoorn PCW (1999b). EXT-mutation analysis and loss of heterozygosity in sporadic and hereditary osteochondromas and secondary chondrosarcomas. Am J Hum Genet 65:689-698.

Bovée JVMG, Van den Broek LJCM, De Boer WI, and Hogendoorn PCW (1998). Expression of growth factors and their receptors in adamantinoma of long bones and the implications for its histogenesis. J Pathol 184:24-30.

Bovée JVMG, Van der Heul RO, Taminiau AHM, and Hogendoorn PCW (1999c). Chondrosarcoma of the Phalanx: A locally aggressive lesion with minimal metastatic potential. A report of 35 cases and a review of the literature. Cancer 86:1724-1732.

De-Boer WI, Vermeij M, Gil Diez de Medina S, Bindels E, Radvanyi F, Van der Kwast TH, and Chopin D (1996). Functions of fibroblast and transforming growth factors in primary organoid-like cultures of normal human urothelium. Lab Invest 75:147-156.

Detre S, Saccani Jotti G, and Dowsett M (1995). A "quickscore" method for immunohistochemical semiquantitation: Validation for oestrogen receptor in breast carcinomas. J Clin Pathol 48:876-878.

Elkhuizen PHM, Voogd AC, Van den Broek LJCM, Tan ITC, Van Houwelingen HC, Leer J-WH, and Van de Vijver MJ (1999). Risk factors for local recurrence after breastconserving therapy for invasive carcinomas: A case-control study of histological factors and alterations in oncogene expression. Int J Radiat Oncol Biol Phys 45:73-83.

Erlebacher A, Filvaroff EH, Gitelman SE, and Derynck R (1995). Toward a molecular understanding of skeletal development. Cell 80:371-378. 
Evans HL, Ayala AG, and Romsdahl MM (1977). Prognostic factors in chondrosarcoma of bone. A clinicopathologic analysis with emphasis on histologic grading. Cancer 40:818-831.

Geirnaerdt MJA, Hermans J, Bloem JL, Kroon HM, Pope TL, Taminiau AHM, and Hogendoorn PCW (1997). Usefulness of radiography in differentiating enchondroma from central grade I chondrosarcoma. AJR Am J Roentgenol 169:1097-1104.

Goldfarb M (1996). Functions of fibroblast growth factors in vertebrate development. Cytokine Growth Factor Rev 7:311325.

Hecht JT, Hogue D, Wang Y, Blanton SH, Wagner M, Strong LC, Raskind W, Hansen MF, and Wells D (1997). Hereditary multiple exostoses (EXT): Mutational studies of familial EXT1 cases and EXT-associated malignancies. Am J Hum Genet 60:80-86.

Hughes SE (1997). Differential expression of the fibroblast growth factor receptor (FGFR) multigene family in normal human adult tissues. J Histochem Cytochem 45:1005-1019.

Hughes SE and Hall PA (1993). Immunolocalization of fibroblast growth factor receptor 1 and its ligands in human tissues. Lab Invest 69:173-182.

Huvos AG (1991). Bone tumors. Diagnosis, treatment, and prognosis. Philadelphia: WB Saunders, 343-395.

Iwamoto M, Shimazu A, Nakashima K, Suzuki F, and Kato $Y$ (1991). Reduction of basic fibroblasts growth factor receptor is coupled with terminal differentiation of chondrocytes. J Biol Chem 266:461-467.

Kato $Y$ and Iwamoto M (1990). Fibroblast growth factor is an inhibitor of chondrocyte terminal differentiation. J Biol Chem 265:5903-5909.

Kitagawa H, Shimakawa H, and Sugahara K (1999). The tumor suppressor EXT-like gene EXTL2 encodes an alpha1, 4-N-acetylhexosaminyltransferase that transfers $\mathrm{N}$-acetylglucosamine to the common glycosaminoglycanprotein linkage region. J Biol Chem 274:13933-13937.

Lind T, Tufaro F, McCormick C, Lindahl U, and Lidholt $\mathrm{K}$ (1998). The putative tumor suppressors EXT1 and EXT2 are glycosyltransferases required for the biosynthesis of heparan sulfate. J Biol Chem 273:26265-26268.

McCormick C, Duncan G, Goutsos KT, and Tufaro F (2000). The putative tumor suppressors EXT1 and EXT2 form a stable complex that accumulates in the golgi apparatus and catalyzes the synthesis of heparan sulfate. Proc Natl Acad Sci USA 97:668-673.

McCormick C, Duncan G, and Tufaro F (1999). New perspectives on the molecular basis of hereditary bone tumours. Mol Med Today 5:481-486.

McCormick C, Leduc Y, Martindale D, Mattison K, Esford LE, Dyer AP, and Tufaro F (1998). The putative tumour suppressor EXT1 alters the expression of cell-surface heparan sulfate. Nat Genet 19:158-161.

Mirra JM, Gold R, Downs J, and Eckardt JJ (1985). A new histologic approach to the differentiation of enchondroma and chondrosarcoma of the bones. A clinicopathologic analysis of 51 cases. Clin Orthop (December):214-237.

Mulder JD, Schütte HE, Kroon HM, and Taconis WK (1993). Radiologic atlas of bone tumors. Amsterdam: Elsevier, 139193.
Naski MC, Colvin JS, Coffin JD, and Ornitz DM (1998). Repression of hedgehog signaling and BMP4 expression in growth plate cartilage by fibroblast growth factor receptor 3 . Development 125:4977-4988.

Qu Z, Huang X-N, Ahmadi P, Andresevic J, Planck SR, Hart CE, and Rosenbaum JT (1995). Expression of basic fibroblast growth factor in synovial tissue from patients with rheumatoid arthritis and degenerative joint disease. Lab Invest 73:339-346.

Reed JA, Albino AP, and McNutt NS (1995). Human cutaneous mast cells express basic fibroblast growth factor. Lab Invest 72:215-222.

Sahni M, Ambrosetti D, Mansukhani A, Gertner R, Levy D, and Basilico C (1999). FGF signaling inhibits chondrocyte proliferation and regulates bone development through the STAT-1 pathway. Genes Dev 13:1361-1366.

Schmale GA, Conrad EU, and Raskind WH (1994). The natural history of hereditary multiple exostoses. J Bone Joint Surg Am 76A:986-992.

Simmons AD, Musy MM, Lopes CS, Hwang L, Yang Y, and Lovett M (1999). A direct interaction between EXT proteins and glycosyltransferases is defective in hereditary multiple exostoses. Hum Mol Genet 8:2155-2164.

Springfield DS, Gebhardt MC, and McGuire MH (1996). Chondrosarcoma: A review. J Bone Joint Surg Am 78A:141-149.

Stickens D, Clines G, Burbee D, Ramos P, Thomas S, Hogue D, Hecht JT, Lovett M, and Evans GA (1996). The EXT2 multiple exostoses gene defines a family of putative tumour suppressor genes. Nat Genet 14:25-32.

The I, Bellaiche Y, and Perrimon N (1999). Hedgehog movement is regulated through tout velu-dependent synthesis of a heparan sulfate proteoglycan. Mol Cell 4:633-639.

Toyoda H, Kinoshita-Toyoda A, and Selleck SB (2000). Structural analysis of glycosaminoglycans in drosophila and caenorhabditis elegans and demonstration that tout-velu, a drosophila gene related to EXT tumor suppressors, affects heparan sulfate in vivo. J Biol Chem 275:2269-2275.

Unni KK (1996). Chondrosarcoma (primary, secondary, dedifferentiated, and clear cell). In: Unni KK, editor. Dahlin's bone tumors. General aspects and data on 11,087 cases. Philadelphia: Lippincott-Raven Publishers, 71-108.

Uria JA, Balbin M, Lopez JM, Alvarez J, Vizoso F, Takigawa M, and Lopez-Otin C (1998). Collagenase-3 (MMP-13) expression in chondrosarcoma cells and its regulation by basic fibroblast growth factor. Am J Pathol 153:91-101.

Van der Eerden BCJ, Karperien M, Gevers EF, Lowik CWGM, and Wit JM (2000). Expression of Indian hedgehog, parathyroid hormone-related protein, and their receptors in the postnatal growth plate of the rat: Evidence for a locally acting growth restraining feedback loop after birth. J Bone Miner Res 15:1045-1055.

Wicklund LC, Pauli RM, Johnston D, and Hecht JT (1995). Natural history study of hereditary multiple exostoses. Am J Med Genet 55:43-46. 
Wuyts W, Van Hul W, Wauters J, Nemtsova M, Reyniers E, Van Hul E, De Boulle K, De Vries BBA, Hendrickx J, Herrygers I, Bossuyt P, Balemans W, Fransen E, Vits L, Coucke P, Nowak NJ, Shows TB, Mallet L, Van den Ouweland AMW,
McGaughran J, Halley DJJ, and Willems P (1996). Positional cloning of a gene involved in hereditary multiple exostoses. Hum Mol Genet 5:1547-1557. 